\title{
The maxillary lateral incisor in the rehabilitation of cleft lip and palate
}

\section{Abstract}

Guida Paola Genovez TEREZA ${ }^{1}$

Marcos Antônio Corrêa dos SANTOS ${ }^{1}$

Vivian Patricia Saldias Vargas

WINCKLER $^{1}$

Ana Lúcia Pompeia Fraga de

ALMEIDA $^{2}$

Gisele da Silva DALBEN ${ }^{1}$
Submitted: March 21, 2017 Modification: June 2, 2017 Accepted: July 16, 2017
Objective: This study analyzed the maintenance of lateral incisors in the dental rehabilitation of individuals with cleft lip and palate. Material and Methods: The study was conducted on a tertiary craniofacial center and comprised retrospective analysis of panoramic and periapical radiographs of Caucasoid individuals with non-syndromic complete unilateral cleft lip and palate, analyzing all radiographs available on the records of each individual, from the first to the last up to 12 years of age. Overall, 2,826 records were reviewed to achieve a sample of 1,000 individuals. Among these, 487 individuals presented the permanent lateral incisors on both cleft and non-cleft sides, which were included in this study. Results: The results were evaluated in percentages and by descriptive statistics. The association between maintenance of the lateral incisor and timing of alveolar bone graft were analyzed by the t test. Among the 487 individuals, 265 had not completed treatment, 62 presented insufficient information, and 44 concluded the treatment elsewhere. Among the remaining 116 individuals, the lateral incisor was extracted from 88 (75.86\%) of them on the cleft side (CS) and from $23(19.83 \%)$ people on the non-cleft side (NCS). The age at accomplishment of alveolar bone graft was significantly associated with maintenance of the lateral incisor on the cleft side $(p<0.01)$. Most extractions were indicated because of the inadequate positioning on the CS and for midline correction on the NCS. Rehabilitation was primarily completed by orthodontic movement (53 individuals on the CS and 13 individuals on the NCS). Conclusion: In conclusion, the lateral incisor on the cleft side was not maintained in most individuals. Positive relationship was observed between extraction of the lateral incisor and age at accomplishment of the alveolar bone graft, suggesting the need to anticipate the initial radiographic evaluation to enhance its maintenance and reduce the procedures required for rehabilitation.

Keywords: Incisor. Cleft lip. Cleft palate. Oral rehabilitation.

'Universidade de São Paulo, Hospital de Reabilitação de Anomalias Craniofaciais, Bauru, São Paulo, Brasil.

${ }^{2}$ Universidade de São Paulo, Faculdade de Odontologia de Bauru, Departamento de Prótese; Hospital de Reabilitação de Anomalias Craniofaciais, Bauru, São Paulo, Brasil.
Corresponding address: Rua Sisele da Silva Dalben 17012-900 Phone: $+55(14)$ 3235-8141 Fax: $+55(14) 3234-7818$ e-mail gsdalben@usp.br 


\section{Introduction}

Individuals with cleft lip and palate present complex skeletal deformities and are subjected to a treatment load that requires several procedures, which begin in childhood and continue up to adulthood, aiming to restore the normal morphology and function.

The treatment of alveolar defects usually requires alveolar bone graft ${ }^{21}$. Even though the alveolar bone graft is widely accepted by professionals for cleft treatment, there is still no consensus concerning the technique, timing and donor site ${ }^{13}$. This procedure was used in the 1960s in an early and primary manner, aiming to stabilize the premaxilla, allow tooth eruption in the cleft area and increase the alar base ${ }^{18}$. Unfortunately, the long-term follow-up revealed severe interferences in maxillary growth and frequent need for procedures of secondary alveolar bone $\operatorname{graft}^{10,15}$. Thus, the secondary bone graft was introduced into alveolar defects of individuals in the mixed dentition stage before eruption of the permanent canine, aiming to minimize late complications ${ }^{1}$. However, recently, it was demonstrated that earlier accomplishment of bone graft, in the deciduous or early mixed dentition, might support the eruption of the lateral incisor $8,14,19$. The results of studies on early bone graft demonstrated favorable graft healing without interference in maxillary growth ${ }^{8,11,19}$; additionally, when the graft is performed to facilitate the eruption of the lateral incisor, the cleft space may be orthodontically repaired in $100 \%$ of individuals ${ }^{8}$.

Despite the high success rates reported in the literature for the secondary alveolar bone graft, there are controversies concerning the age of accomplishment, suggesting the need to establish a specific treatment protocol ${ }^{2,9}$. However, we believe no study has demonstrated the utilization of lateral incisors in the dental rehabilitation of individuals with cleft lip or palate. The lateral incisor is directly related to the rehabilitation of these individuals, and the knowledge of its impact is fundamental to develop effective treatment protocols while minimizing the burden of care. This study analyzed (1) the prevalence of extraction of maxillary lateral incisors, (2) the reasons for extraction indication, (3) the association between maintenance of the lateral incisor on the cleft side and age at accomplishment of the alveolar bone graft; and (4) the types of treatment delivered for dental rehabilitation of individuals with cleft lip and palate.

\section{Material and methods}

This study was approved by the Institutional Review Board of HRAC/USP (protocol no. 241/2011).

The inclusion criteria were: 1 ) Caucasoid individuals with non-syndromic complete unilateral cleft lip and palate, 2) presence of panoramic and periapical radiographs in the individual's files, from the first radiographs obtained up to the last up to 12 years of age, in addition to thorough dental history in the records to analyze the presence or absence of the permanent lateral incisors on the cleft and non-cleft sides, 3) individuals originally presenting permanent lateral incisors on both cleft and non-cleft sides.

A single examiner reviewed 2,826 records of individuals with non-syndromic complete unilateral cleft lip and palate, regularly registered in the institution. Among these individuals, we selected those whose records contained panoramic and periapical radiographs from the first to the last up to 12 years of age, which led to a sample of 1,000 individuals. Among these, an additional selection was performed to include only individuals whose radiographs available and dental history allowed reliable analysis of the presence or absence of permanent lateral incisors on both cleft and non-cleft sides. This led to a sample of 487 individuals who presented the permanent lateral incisors on the cleft and non-cleft sides, who were included in this study (Figure 1).

The authors did not find any study analyzing the extraction of lateral incisors for orthodontic/dental rehabilitation. For this reason, sample size calculation could not be performed. Thus, after study completion, the post-hoc power of the study was calculated using the following parameters: sample size 487 individuals/ percentage of extraction of lateral incisors in this sample $76 \%$ /putative percentage of extraction of lateral incisors in the overall population $1 \%$ /alpha error of 0.05 , which revealed a post-hoc power of $100 \%$.

This study comprised retrospective analysis of such records, searching for information about maintenance of the permanent lateral incisor on the cleft and noncleft sides for completion of dental rehabilitation. In cases with indication of lateral incisor extraction, information about the specialist who indicated the extraction and the reason for such decision was 
Non-syndromic

complete

unilateral cleft

lip and palate
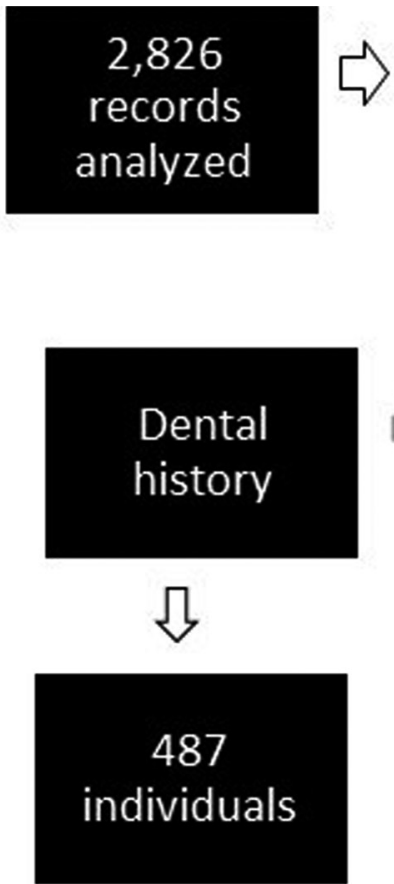

1,000

individuals with

complete

radiographs
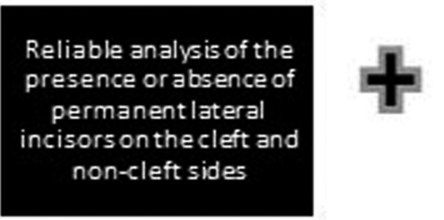

achieved, in addition to the treatment indicated for space closure in the cleft region. Secondarily, the study also evaluated the association between maintenance of the lateral incisor on the cleft side and age at accomplishment of alveolar bone graft, using the t test, at a significance level of $p<0.05$. The other results were evaluated in percentages and by descriptive statistics.

\section{Results}

Among the 487 individuals previously selected who presented the permanent lateral incisor on both cleft and non-cleft sides, 265 individuals had not completed the dental rehabilitation until the onset of this study, 62 individuals exhibited insufficient information on the records, and 44 completed the treatment elsewhere. Thus, the other analyses were conducted on a sample of 116 individuals with complete unilateral cleft lip and palate, with presence of one permanent lateral incisor on both cleft and non-cleft sides, and dental rehabilitation concluded before the onset of this study.

The lateral incisor on the cleft side was maintained in 28 individuals (24.14\%), while the lateral incisor on the non-cleft side was maintained in 93 individuals $(80.17 \%)$.

The mean age at accomplishment of alveolar bone graft was 14.30 years ( $S D=4.63)$, ranging from 8 to 30 years. Statistically significant association was found between age at bone graft and maintenance of the lateral incisor only for the cleft side (Table 1 ).

In individuals submitted to extractions, the specialist indicating them was informed only on the records of 26 individuals, with predominance of orthodontists (21), followed by maxillofacial surgeons (4) and prosthodontist (1). The reason for extraction indication was described in 16 cases (Table 2), including inadequate positioning (13), facilitation of orthodontic mechanics (1), lack of space in the dental arch (1) and lack of periodontal support (1). In cases with extraction of the lateral incisor, the corresponding space was rehabilitated by orthodontic movement with mesial movement of the canine (53), fixed prosthesis (17) and dental implant (10), without information available for the other eight individuals.

The specialist who indicated the extraction of the lateral incisor on the non-cleft side was described in the records of 13 individuals, all of which were indicated by the orthodontist. The reason for extraction indication was described in nine cases, including midline correction (4), inadequate positioning (3) and achievement of space at the posterior region (2). In cases of extraction of the lateral incisor, the corresponding space was rehabilitated by orthodontic movement with mesial movement of the canine (13) and fixed prosthesis (1), without information available for the other nine individuals. 
Table 1- Maintenance of lateral incisor according to the age at accomplishment of alveolar bone graft

\begin{tabular}{ccccc}
\hline $\begin{array}{c}\text { Maintenance of lateral } \\
\text { incisor }\end{array}$ & \multicolumn{4}{c}{ Age at accomplishment of alveolar bone graft } \\
Cleft side & $\mathbf{n}$ & Mean & Standard deviation & p \\
\hline Yes & 28 & 12.25 & 2.43 & $<0.01^{*}$ \\
No & 88 & 14.91 & 4.97 & $\mathrm{p}$ \\
Non-cleft side & $\mathrm{n}$ & Mean & Standard deviation & $0.73^{* *} \mathrm{~ns}$ \\
\hline Yes & 93 & 14.19 & 4.48 & 5.26 \\
\hline No & 23 & 14.57 & & \\
\hline
\end{tabular}

${ }^{*} \mathrm{t}$ test with Welch correction; ${ }^{* *} \mathrm{t}$ test

Table 2- Reasons for the indication of lateral incisor extraction

\begin{tabular}{ccc}
\hline Reasons for indication of extraction & Cleft side & Non-cleft side \\
\hline Inadequate positioning & 13 & 3 \\
\hline Facilitation of orthodontic movement & 1 & 2 \\
\hline Lack of space in the dental arch & 1 & 4 \\
\hline Lack of periodontal support & 1 & 14 \\
\hline Midline correction & 72 & \\
\hline No information & & \\
\hline
\end{tabular}

Table 3- Treatment performed for dental rehabilitation

\begin{tabular}{lcc}
\hline \multicolumn{1}{c}{ Treatment } & Cleft side & Non-cleft side \\
\hline Mesial movement of canine & 53 & 13 \\
\hline Dental prosthesis & 17 & 1 \\
\hline Dental implant & 10 & - \\
No information & 8 & 9 \\
\hline
\end{tabular}

\section{Discussion}

The rehabilitation of individuals with complete unilateral cleft lip and palate is complex and involves several stages, including alveolar bone graft, which is fundamental to join the alveolar segments ${ }^{1}$. The main objectives of alveolar reconstruction are closure of nasal fistula, unification of maxillary segments, providing bone support for eruption of anterior teeth and for the nasal base, and allow prosthetic reconstruction including dental implants ${ }^{1,3,5}$.

Bone graft should be performed whenever possible to facilitate the eruption of teeth close to the cleft or orthodontic movement, either of the canine or lateral incisor ${ }^{8}$. Most studies consider the roots of canines to establish the timing for accomplishment of bone graft, considering one fourth and/or two thirds of its length ${ }^{4,12,16,21}$. However, recent studies demonstrated the possibility of alveolar bone graft in the deciduous dentition, providing sufficient bone support for eruption of central and lateral incisors with more favorable positioning of maxillary teeth ${ }^{20}$. Also, orthodontic space closure may be possible in $100 \%$ of cases when the bone graft is performed to facilitate the eruption of lateral incisor 7,8 . Additionally, individuals older than 12 years are four times more likely to present postoperative complications after alveolar bone graft ${ }^{13}$. Recently, the accomplishment of alveolar graft before eruption of the lateral incisor reduced the frequency of permanent canine impaction. This intervention does not change the risk, even though it was smaller in the group submitted to early bone graft ${ }^{6}$.

The eruption and adequate positioning of the permanent lateral incisor in the cleft area maintains the bone graft by mechanical stimulation, but also achieves stable functional occlusion, allowing normal maxillary growth and more harmonious facial and dental esthetics ${ }^{22}$.

In this study, the mean age at accomplishment of alveolar bone graft was 14.30 years $(S D=4.63)$, ranging from 8 to 30 years. This may have been influenced by the continental dimensions of the country and predominantly low socioeconomic status of the population assisted at the institution; and this may have contributed to the low percentage of maintenance of the lateral incisor on the cleft side, as demonstrated by the statistically significant association between maintenance of the lateral incisor and age at accomplishment of alveolar bone graft (Table 1). However, this is the first study on this subject conducted at the institution and the 
findings should be carefully interpreted. Additionally, the presence, position and root morphology of the lateral incisor should be carefully analyzed to assure the possibility of maintenance of this tooth ${ }^{18}$. Thus, ideally, the maxillofacial surgeon should evaluate both the individual and the radiographs before the mixed dentition stage to properly determine the better timing for bone graft, thus allowing more favorable positioning of the lateral incisor ${ }^{11}$.

Our results revealed indication of 17 prostheses and 10 implants, and the space corresponding to the maxillary lateral incisor on the cleft side was closed by orthodontic mesial movement in 53 individuals (Table 3). When necessary and possible, the mesial movement of canine is favorable to reduce the utilization of prostheses and implants. Most lateral incisors on the non-cleft side were maintained. The accomplishment of bone graft before eruption of the incisors provides bone support for the eruption of these teeth, restoring the maxillary arch shape and enhancing their retention and gingival health. The burden of care may be reduced by minimizing the treatment stages and allowing earlier treatment completion, eliminating the need for rehabilitation with prostheses in adulthood ${ }^{11,17}$ and providing more favorable esthetic results by closing the space with a natural tooth. This aspect highlights the importance of bone graft and orthodontics in individuals with the lateral incisor.

Unfortunately, several records were incompletely filled by the different professionals treating the individuals in the institution. This is an inherent limitation of retrospective studies, especially those conducted in large institutions involving different specialists, such as in this case.

Based on these results, the authors concluded that 1) most of the lateral incisors (76\%) adjacent to the cleft were extracted; 2 ) the main reason for extracting the lateral incisor was its inadequate position; 3 ) the earlier the bone grafting procedure is accomplished, the greater are the chances of maintaining the lateral incisor; and 4) the rehabilitation of the cleft area in most of the cases was achieved by means of orthodontic space closure.

Thus, analyzing the maintenance of lateral incisor for dental rehabilitation of individuals with complete unilateral cleft lip and palate, we observed that evaluation for alveolar bone graft is mostly performed considering the canine, i.e. when the lateral incisor is already erupted, thus missing the ideal timing of bone graft for this tooth, despite present and in good conditions. Considering the reports of success of secondary alveolar graft performed before eruption of the lateral incisor and the low rate of use of this tooth when bone graft is performed after eruption of the lateral incisor, as demonstrated in this study, the possibility of a slight anticipation in the timing of alveolar bone graft might be considered to increase the possibility of utilization of the lateral incisor. These findings suggest the need to customize the timing of orthodontic evaluation and alveolar bone graft in individuals with lateral incisor in the cleft area, considering the possibility of its eruption through the bone graft to increase the maintenance of the lateral incisor, when present, and reduce the burden of care for dental rehabilitation of individuals with cleft lip and palate.

\section{Conclusion}

The lateral incisor on the cleft side was not maintained in most individuals. There was positive relationship between extraction of the lateral incisor and age at accomplishment of the alveolar bone graft, suggesting the need to anticipate the initial radiographic evaluation to enhance its maintenance and reduce the steps required for rehabilitation.

\section{Acknowledgement}

The authors thank FAPESP for funding this study (research grant 2010/14481-2).

\section{References}

1- Boyne PJ, Sands NR.Combined orthodontic-surgical management of residual palato-alveolar cleft defects. Am J Orthod. 1976;70(1):20-37. 2- Freihofer HP, Borstlap WA, Kuijpers-Jagtman AM, Voorsmit RA, van Damme PA, Heidbüchel $\mathrm{KL}$, et al. Timing and transplant materials for closure of alveolar clefts: a clinical comparison of 296 cases. J Cranio Maxillofac Surg. 1993;21(4):143-8.

3- Freitas JA, Almeida AL, Soares S, Neves LT, Garib DG, TrindadeSuedam IK, et al. Rehabilitative treatment of cleft lip and palate: experience of the Hospital for Rehabilitation of Craniofacial Anomalies/ USP (HRAC/USP) - Part 4: Oral Rehabilitation. J Appl Oral Sci. 2013;21(3):284-92

4- Hynes PJ, Earley MJ. Assessment of secondary alveolar bone grafting using a modification of the Bergland grading system. Br J Plastic Surg. 2003;56(7):630-6. 
5- Kearns G, Perrott DH, Sharma A, Kaban LB, Vargervik K. Placement of endosseous implants in grafted alveolar clefts. Cleft Palate Craniofac

J. 1997;34(6):520-5.

6- Kleinpoort F, Ferchichi H, Belkhou A, Tramini P, Bigorre M, Captier G. Early secondary bone grafting in children with alveolar cleft does not modify the risk of maxillary permanent canine impaction at the age of 10 years. J Craniomaxillofac Surg. 2017;45(4):515-9.

7- Lilja J. Alveolar bone grafting. Indian J Plast Surg. 2009;42 Suppl:S110-5

8- Lilja J, Kalaaji A, Friede H, Elander A. Combined bone grafting and delayed closure of the hard palate in patients with unilateral cleft lip and palate: facilitation of lateral incisor eruption and evaluation of indicators for timing of the procedure. Cleft Palate Craniofac J. 2000;37(1):98-105.

9- Luque-Martín E, Tobella-Camps ML, Rivera-Baró A. Alveolar graft in the cleft lip and palate patient: review of 104 cases. Med Oral Patol Oral Cir Bucal. 2014;19(5): e531-7.

10- Matic DB, Power SM. Evaluating the success of gingivoperiosteoplasty versus secondary bone grafting in patients with unilateral clefts. Plast Reconstr Surg. 2008;121(4):1343-53.

11- Miller LL, Kauffmann D, St John D, Wang D, Grant JH, Waite PD. Retrospective review of 99 patients with secondary alveolar cleft repair. J Oral Maxillofac Surg. 2010;68(6):1283-9.

12- Ochs MW. Alveolar cleft bone grafting (Part II): Secondary bone grafting. J Oral Maxillofac Surg. 1996;54(1):83-8.

13- Pessoa EA, Braune A, Casado PL, Tannure PN. Alveolar bone graft: clinical profile and risk factors for complications in oral cleft patients. Cleft Palate Craniofac J. 2017;54(5):530-4.
14- Precious DS. Alveolar bone grafting at 6 years of age. J Oral Maxillofac Surg. 2009;67(10):2045-53.

15- Rehrmann AH, Koberg WR, Koch $\mathrm{H}$. Long-term postoperative results of primary and secondary bone grafting in complete clefts of the lip and palate. Cleft Palate J. 1970;7:206-21.

16- Seifeldin SA. Is alveolar cleft reconstruction still controversial? (Review of literature). Saudi Dent J. 2016;28(1):3-11.

17- Shashua D, Omnell ML. Radiographic determination of the position of the maxillary lateral incisor in the cleft alveolus and parameters for assessing its habilitation prospects. Cleft Palate Craniofac J. 2000;37(1):21-5

18- Skoog T. The use of periosteal flaps in the repair of clefts of the primary palate. Cleft Palate J. 1965;2:332-9.

19- Talmant JC, Lumineau JP, Rousteau G. Cleft lip, maxilla and palate treatment by Dr. Talmant's team in Nantes. Ann Chir Plast Esthet. 2002;47(2):116-25.

20- Touzet-Roumazeille S, Vi-Fane B, Kadlub N, Genin M, Dissaux $C$, Raoul G, et al. Osseous and dental outcomes of primary gingivoperiosteoplasty with iliac bone graft: a radiological evaluation. J Craniomaxillofac Surg. 2015;43(6):950-5.

21- Trindade IK, Mazzottini R, Silva Filho OG, Trindade IE, Debon $M C$. Long-term radiographic assessment of secondary alveolar bone grafting outcomes in patients with alveolar clefts. Oral Surg Oral Med Oral Pathol Oral Radiol Endod. 2005;100(3):271-7.

22- Witsenburg $B$. The reconstruction of anterior residual bone defects in patients with cleft lip, alveolus and palate. A review. J Maxillofac Surg. 1985;13(5):197-208. 